\title{
Installing and Running Telescopes on Skynet for Research and Instruction
}

\author{
Daniel B. Caton ${ }^{1 *}$
}

\begin{abstract} that are discussed.

${ }^{1}$ Appalachian State University

${ }^{*}$ Corresponding author: catondb@appstate.edu
\end{abstract}

We have two telescopes on the Skynet system at our Dark Sky Observatory (DSO), with hopes to put on two more existing telescopes. The experience-basically good-of putting telescopes on Skynet for use by faculty, students, and researchers worldwide is described, as well as some of the difficulties of installation and maintenance, and beneficial experiences running them. Our first telescope, a 14-inch Celestron OTA on a Software Bisque Paramount ME German equatorial mount, was a relatively easy installation given its close match to the original Skynet PROMPT telescopes' configuration. The second installation, a 17-inch PlaneWave OTA on a Mathis fork mount, has proven more challenging. We would like to put our 32-inch at DSO, as well as the campus 16-inch, on Skynet but the integral open-loop dome control in the DFM Engineering Telescope Control System presents some problems

\section{Introduction}

\section{Skynet}

Skynet is an international network of telescopes originally implemented to do quick-response observations of the optical afterglow of Gamma Ray Bursts (GRBs). Hosted at the University of North Carolina-Chapel Hill (UNC-CH), it began with the six PROMPT (Panchromatic Robotic Optical Monitoring and Polarimetry Telescopes) instruments in Chile. These were designed to make simultaneous observations of GRBs in multiple colors to determine the redshift that is measureable from such photometric data (Reichart et al., 2005). Since GRB events use only a small fraction of the available observing time, the Skynet system was designed to not only command the response to GRBs but to have a backbone queue-scheduled observing mode to acquire photometric data on any number of targets for Skynet member institutions. The system was designed generally enough to allow non-PROMPT telescopes to join the Skynet network.

\section{Dark Sky Observatory}

At Appalachian State University's Dark Sky

Observatory, we have two telescopes that operate on Skynet. The first of those, DSO-14, was one of the first non-PROMPT telescopes to operate on Skynet. The second, DSO-17, was installed a few years later. We describe here the experience of implementing these telescopes and operating them on Skynet, from the viewpoint of the telescope owner. This is an overview of that experience, the details of the implementation of DSO-14 have been published by Smith et al. (2016).

\section{DSO-14}

The first telescope, DSO-14, was originally an instructional telescope used manually in a 5-meter diameter dome on the main campus of Appalachian State. A Celestron C14, was provided by a grant from the National Science Foundation, and used by astronomy majors and others in a class in observational techniques. It was originally equipped with an Optec SSP-3 PIN diode 
photometer, and later, at the dawn of inexpensive digital imaging, a Lynxx CCD camera. It was later replaced with a DFM Engineering 16-inch telescope provided by a subsequent NSF grant. The 14-inch Celestron remained in storage until our Skynet project.

In 2007 we decided to have a graduate student, Adam Blythe Smith, implement this telescope on Skynet as his thesis project in our Masters in Applied Physics program (now a Masters in Engineering Physics). Our Masters program has always had an emphasis on electronics and interfacing computers to real-time instrument and experiment control. Adam had participated in a summer internship in 2006 with the Skynet program at UNC-CH and had the knowledge and experience to tackle the project. The C14's optical tube assembly (OTA), was mounted on a

Paramount ME German equatorial mount that was loaned to us by the Skynet team. We later obtained our own Paramount with an NSF Major Research Instrumentation grant. Figure 1 shows Adam and the telescope.

The telescope was placed in a previously existing Ash brand dome that had at one time housed a 16-inch Meade Newtonian telescope mounted on a German Equatorial mount. That setup had been used by department faculty member Joe Pollock, at the end of the era of chemical photography, to take plates of quasars and variable stars. The dome and telescope had gone unused for several years until the campus science building housing the DFM-16 was to be demolished and replaced. For the two years of that construction project, the DFM telescope was moved to DSO and used in this dome. A commercially made (Leonard) outbuilding was attached to the dome to provide a climate-controlled room for the computers and observer (Figure 2).

After the two years (during which Smith was an undergraduate), the DFM was moved back to the campus to the new science building and the DSO dome sat empty. Then Smith started the DSO-14 Skynet project.

The basic configuration needed to comply with

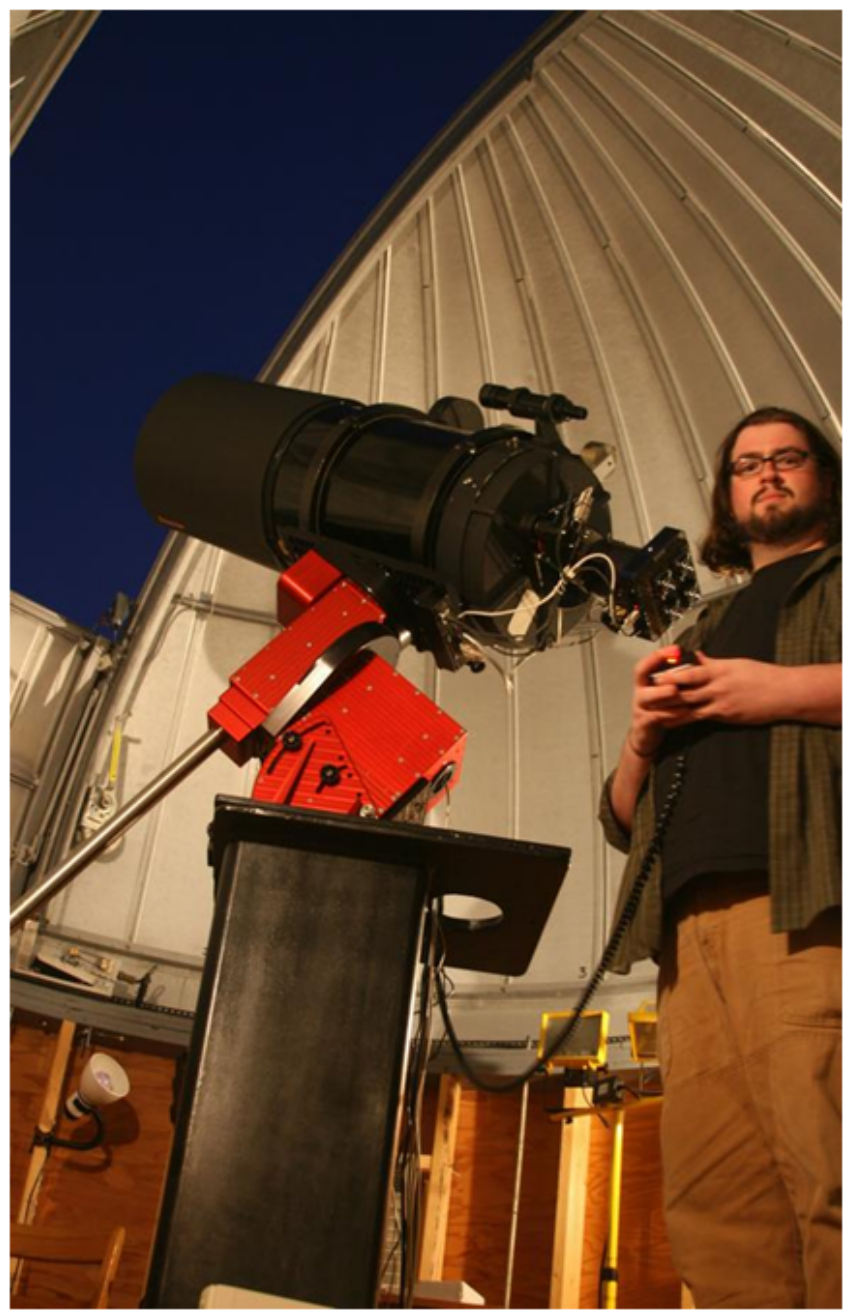

Figure 1. Adam Smith at the DSO-14 telescope.

Skynet requires a telescope on a mount that can be controlled by the program TheSky, sold separately by Software Bisque but also directly controlling their Paramount series mounts. We use Version 6 of TheSky but newer versions are compliant. The CCD camera must be controlled by the program Maxim DL, which also controls the filter wheel. Finally, the operation of the dome must comply by being controlled by either The Sky or an auxiliary program such as AutomaDome. We interfaced our Ash dome to a control system sold by

Observa-Dome Laboratories, via AutomaDome software. (See Smith et al. 2016 for more details on Skynet.)

To untether the dome shutter motors mounted on the rotating dome we installed a pair of deep-cycle marine batteries charged by solar panels mounted 


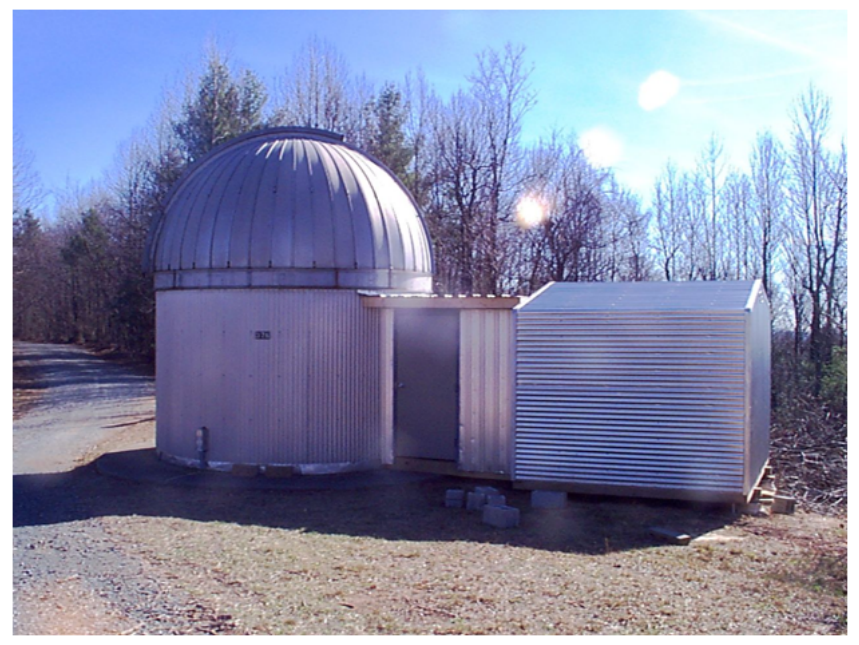

Figure 2. The DSO-14's dome and control room.

on the dome (Figure 3). The control system communicates with the control computer by radio (a watchdog system closes the dome if it loses contact with the computer). Initially, to save money, we used an inverter to convert this to AC to power the originally supplied dome shutter motor. However, the energy budget required to keep it on (to receive commands), was high for these batteries. Finally, on New Year's Eve, December 31, 2009, the dome would not close when commanded, as a blizzard was approaching. The Skynet programmer at that time, Kevin Ivarsen, spent the evening disassembling the dome code to try to find a solution but none was found. The author drove out to DSO, 20 miles from campus, find the inverter had tripped its reset function and the batteries were out of charge. Charging the batteries for a while allowed the dome to be closed, and a military-specification inverter good to well below freezing was obtained. Eventually, a grant allowed us to afford to replace the AC motor with a DC one, eliminating the load of the inverter. Finally, Ivarsen wrote an application to move the dome during the day to keep the solar panels pointing at the sun, for maximum charging (the dome rotation is powered by the line current, so it does not use battery reserve).

The dome system works well with some caveats. After burning out one dome rotation motor we changed the interval of daytime solar tracking to move every 15 degrees (frequent starts are hard on a motor). If there is a cloudy spell of several days the batteries may run down completely. This could be bad if the closure attempt was on bad weather moving in. But, the Observa-Dome system is closed loop: Skynet knows the dome has not closed.

We also found that TheSky6 only tracks the dome on a slew; it does not track the dome with the tracking telescope. We had to install another application, TheSky Link, which was available from Observa-Dome Laboratories, that makes the dome track with the telescope under TheSky6 control.

The telescope was modified some to make it more stable. The C14's primary mirror, the default focusing mechanism, was locked in place to avoid mirror flop with changing telescope orientation. We then first used a JMI electric focuser but later changed to an Optec TCF-S3

Temperature-compensating focuser. This was needed since the Celestron aluminum OTA expands significantly during temperature drops overnight, causing focus changes. The Optec unit is also more robust, can carry a larger load, and has more travel. The focuser is controlled via FocusMax software.

\section{DSO-17}

While the 14-inch telescope implementation was fairly straightforward due to its close resemblance to the original PROMPT telescopes, the 17-inch presented more challenges. This telescope is a 17-inch PlaneWave OTA mounted on a Mathis fork mount. The system is composed of ASCOM drivers and a graphical interface that runs on the control computer. Its SBIG camera is controlled by Maxim DL and the telescope control system is commanded by TheSky, making it nominally compliant with the Skynet standards.

Initial problems included the drive not turning off when Skynet parked the telescope at dawn. The original Astro-Physics telescope control card was replaced with a Sidereal Technology card, allowing more reliable control and support of home 


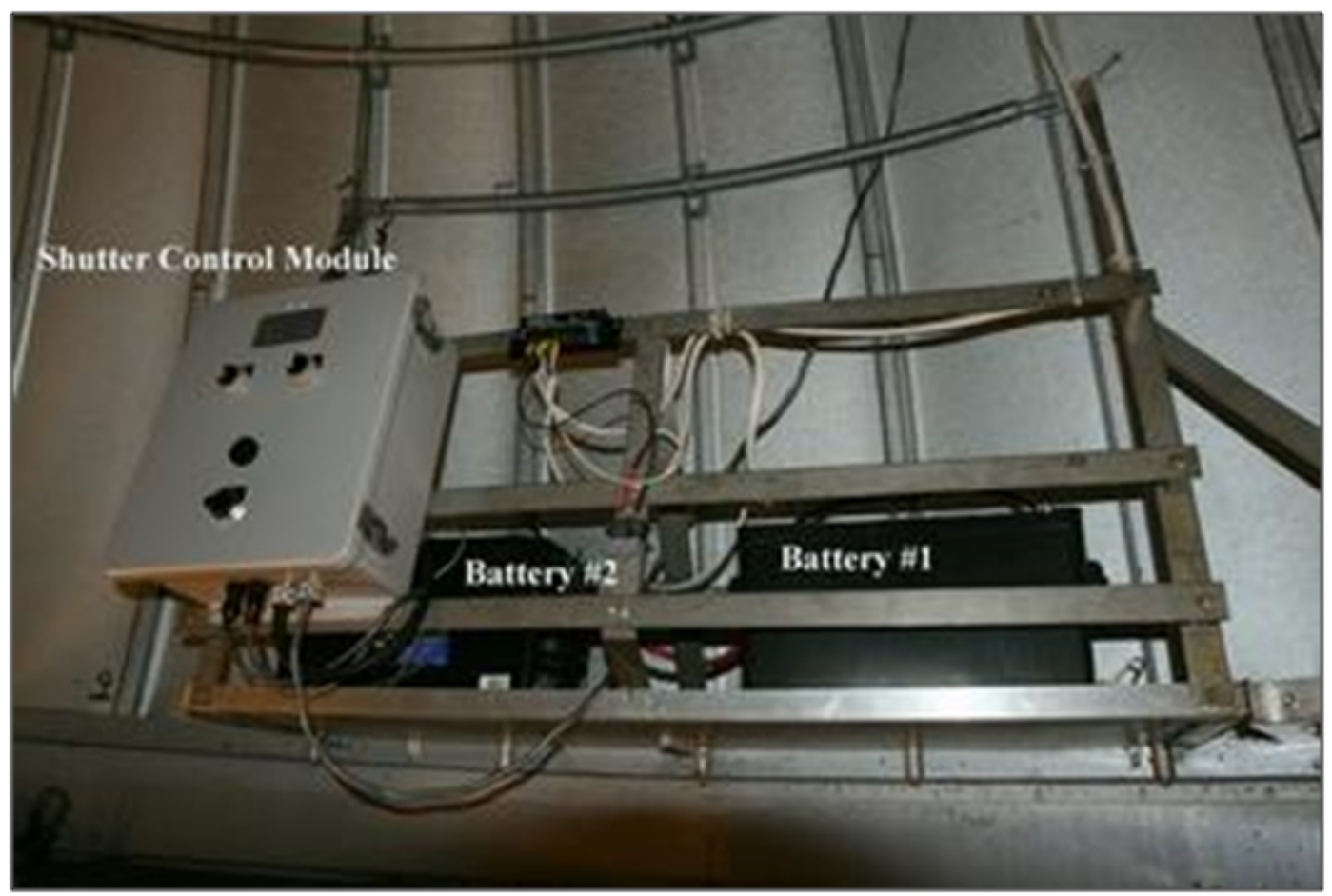

Figure 3. DSO-14's dome shutter control module and solar-charged batteries.

positions. After a good deal of experimenting with the Skynet Terminator program implementation by the Skynet programmer, these problems were overcome. The DSO-17 does not require a temperature-compensating focuser but does have individual focus positions for the various filters. These values have to be determined experimentally and their values entered into Terminator, the client program that is the interface to the Skynet system. Terminator then moves the focus for each exposure, based on the filter.

\section{DFM Telescopes}

There are two more telescopes we would like to implement on Skynet but they present even greater challenges. These are the 32-inch telescope at DSO and a 16-inch on campus. Both were made by DFM Engineering and are of a more robust category than the 14-inch or 17-inch. However, there are complicating factors. While the 14-inch and 17-inch have one computer to control the telescope and camera, the DFMs came with special, rack-mounted industrial computers that run the Telescope Control System (TCS). Maxim DL and TheSky run on a different computer. This presents some challenges although DFM's TCS commands can be received over a network connecting all the computers.

A problem that is more serious is the fact that the DFM TCS controls the dome, and this system is open loop: The TCS does not actually know if the dome reaches close or open position. It merely sends the command to the dome shutter controller, which stops the dome shutter motor with limit switches (these are Observa-Domes and use hardware similar to the other telescopes but do not have the RF link for communication). Further, they do not have watchdog systems to close the dome, or battery power, in the event of a power failure or computer problem. These characteristics present the possibility of the dome not actually closing after a close command is sent upon the arrival of bad weather.

That being said, we already use these DFM telescopes in untended mode, with Boltwood cloud sensors to detect bad weather and directly signal the TCS computer to close the dome. Any number of failures could already lead to a dome not closing before the arrival of rain or snow. It is a risk that 
we just choose to take. We still hope to get these DFM telescopes onto Skynet. That will require custom programming of a version of Terminator for communicating with TCS for dome control, and communication with two computers, since TCS does not run on the computer running Maxim DL.

\section{Support Systems for Skynet Control}

Several systems comprise the environmental monitoring and remote control needed for safe, untended observing, whether on Skynet or simply during untended, remote use.

\section{Webcams and All-Sky Cameras}

While not directly accessed by the Skynet system, we find it essential to have webcams and remote-controlled lights to check the status of the telescopes and domes. Sometimes one needs to check whether the dome is actually open or closed. Plus, it is good to keep an eye on the scope status during the daytime. For these purposes each dome has an ordinary Logitech webcam pointed at the telescope and dome interior. The dome lights are remotely controlled using X10 technology and their ActiveHome computer interface. That system sends commands over the power wires to turn modules and their loads on and off. We uses timers in Active Home to automatically turn the dome light on after dawn and off at dusk. (We also use network-connected power strips to turn devices on and off.)

We use an SBIG All-Sky camera to watch the entire sky day and night (it is monochrome but infrared sensitive, and more sensitive than the color version). This, too, is used manually and is not accessed by the Skynet system. But, it is often important to verify the cloud conditions.

\section{Weather Station, Cloud Sensor, and Weath- erman}

Monitoring weather conditions is essential for both remote and automatic observing. We have a Davis Vantage Pro II weather station that provides weather for remote users but is not the standard used by Skynet. Instead, an application called Weatherman monitors the cloudiness, precipitation, and wind speed reported by a Boltwood cloud sensor. The cloud sensor provides a hardware switch that can be used to directly close domes controlled by both the Observa-Dome systems (the DSO-14 and -17), and the DFM TCS (the 32-inch and campus 16-inch). These control inputs are used on the DFM telescopes, which are not yet on Skynet, for weather protection during automatic, unattended operation. However, it was found that there was a software conflict between the Observa-Dome systems and the control of the dome by Skynet's Terminator client software: simultaneous operation would throw random errors that would require human intervention.

This situation was solved by the Skynet programmer, Kevin Ivarsen, who wrote a program called Weatherman, which monitors the weather via the Boltwood cloud sensor and opens and closes the dome based on conditions. Weatherman can run on any PC at the observatory and controls all domes at the observatory with telescopes on Skynet. Its parameters (cloudiness and wind) are adjustable, a feature needed since we have found that parameter ranges change somewhat seasonally. We have found Weatherman to be very reliable.

\section{The Joys of Skynet}

The author has found the experience of running telescopes on Skynet to be an overall positive experience with certain special joys. There is the benefit of using a well-developed and tuned system to provide automatic operation of one's telescopes. This vastly increases the effective use of the telescopes as well as providing access to other users. In the case of research, the author has provided time to various observing campaigns (e.g. the OJ 287 outburst campaign, Valtonen et al. 2016). By Skynet rules, publications of work that included Skynet observations must include some specific site personnel as well as Skynet team members as co-authors. This is only fair since we spend a significant amount of our professional time developing and maintaining the observatory 
facilities and cannot do as much research as our colleagues who only use the facilities. This has also been rewarding by involving the telescope owners in a diverse range of research areas.

It is fun to provide observing opportunities to students who can experience getting real data (or even just pretty pictures), using a system that does not require them to be up all night. This includes both our own students and students worldwide, including those in the Skynet Junior Scholars program. This also allows these students to get their data with a queue-scheduled, online observing system that is like those increasingly used by professional astronomers.

We have enjoyed contributing to the science of Gamma Ray Bursts, with our scopes responding to a couple of dozen GRB observing requests. This, after all, was the original purpose of Skynet.

There has also been satisfaction with working with the Skynet team and helping contribute to the development of the system. We were one of the first non-PROMPT sites to join and our interaction with the Skynet team led to a better system for all involved. Indeed, the development of Weatherman was needed at other sites like ours, which have much more dynamic and wet weather than found at the original PROMPT scopes in Chile.

\section{Frustrations and Laments}

Of course, any ambitious system like this as well as local problems will cause some frustrating problems as well as unmet desires.

The main one is that having a telescope on Skynet is like owning a cow-you have to milk it every day whether you want to or not. That is, things break, software has glitches, and one has to keep an eye on its operation every night to be sure it is working smoothly. In such a complex system, one will occasionally get errors that you may never be able to understand well enough to fix (if possible at all). Sometimes the dome does not open or does not track the target. We get camera errors occasionally that may be due to errors in the USB extenders (or not). If the system locks up and it is critical to an observer, you may get emails. Indeed, Skynet itself can email the telescope owner when certain errors occur. However, Skynet does protect the telescope and in the event of critical problems, the telescope and dome go into a safe mode until the problem is resolved.

We do lament that we have not yet been able to get our DFM telescopes on Skynet since there are good opportunities to have those respond to calls for a GRB or, now, a kilonova, and other gravitational events reported by LIGO and/or Virgo, or a neutrino event alert from IceCube. In addition, the campus DFM-16 has a lot of available time since it is not used by students very late or on weekends. We still hope to accomplish getting these telescopes on the system.

Finally, we recognize that with the development of more automatic, queue-scheduled, remote-use or big-data-stream observing (like LSST), the observer is increasingly distanced from being under the night sky. The romance is gone. While those of us who used to observe in the cold can really appreciate these new modes, we discovered astronomy in the dark, under the magic of the stars. Will our newer generations of students find the new modes as attractive? The author has had several students who want to do the old-school observing, up all night. While some of my work includes such observing and I offer opportunities to experience it with me, I have to tell them they were born a few decades too late.

\section{Conclusions}

The experience of implementing and running Skynet telescopes has been overall very good, and we can recommend any one who has a Skynet-compliant telescope to consider putting it on the system. One does need to be technically knowledgeable and be up to the challenges of integrating a few hardware and software systems, but the Skynet team will do much of the software work, remotely. 


\section{Acknowledgements}

The author would like to thank Lee Hawkins, our Observatory Engineer, for developing and maintaining the telescopes and domes at the Dark Sky Observatory. We are grateful for the support of the National Science Foundation, NASA Space Grant, and the Appalachian State University Research Council for their support of these projects.

\section{References}

Reichart, D., Nysewander, M., Moran, J., Bartelme, J., Bayliss, M., Foster, A., Clemens, J., Price, P., Evans, C., Salmonson, J., et al. (2005). PROMPT: Panchromatic robotic optical monitoring and polarimetry telescopes. Nuovo Cimento della Societa Italiana di Fisica C, 28(4-5):767-770.

Smith, A. B., Caton, D. B., and Hawkins, R. L. (2016). Implementation and Operation of a Robotic Telescope on Skynet. Publications of the Astronomical Society of the Pacific, 128(963):055002.

Valtonen, M., Zola, S., Ciprini, S., Gopakumar, A., Matsumoto, K., Sadakane, K., Kidger, M., Gazeas, K., Nilsson, K., Berdyugin, A., et al. (2016). Primary black hole spin in OJ 287 as determined by the general relativity centenary flare. The Astrophysical Journal Letters, 819(2):L37. 